\title{
A Qualitative Study of Home-Based and Hybrid Cardiac Rehabilitation During COVID-19
}

Smitha Ganeshan ( $\nabla$ smitha.ganeshan@ucsf.edu )

University of California, San Francisco

Crystal Tse

University of California, San Francisco

Alexis Beatty

University of California, San Francisco

\section{Research Article}

Keywords: Cardiac rehabilitation, Secondary Prevention, telehealth

Posted Date: February 12th, 2021

DOI: https://doi.org/10.21203/rs.3.rs-151295/v1

License: (1) This work is licensed under a Creative Commons Attribution 4.0 International License. Read Full License 


\section{Abstract}

\section{Background:}

Because of the need for distancing during the COVID-19 pandemic, many cardiac rehabilitation (CR) centers suspended in-person services and pivoted to home-based CR (HBCR). In this study, we qualitatively evaluated implementation of HBCR, which included weekly phone or video visits for individualized exercise plans, nutrition and health education counseling, wellness sessions, and optional mobile phone applications.

\section{Methods:}

Patient participants and staff members (physician, nurses, exercise physiologists, dietician, administrative staff) participated in semi-structured interviews. Two independent reviewers coded interview transcripts for emergent themes and pre-specified themes from the Theory of Planned Behavior, Unified Theory of Acceptance and Use of Technology, and the Consolidated Framework for Implementation Research.

\section{Results:}

A total of 12 patients and 7 staff were interviewed. Narrative descriptions highlighted the isolation, fear, and disruption of life activities during COVID-19. Key facilitators of the HBCR patient experience included strong relationships with staff who served as coaches and sources of accountability and the ability of HBCR to deliver an individually tailored experience within a patient's home. Important organizational factors for implementation included leadership buy-in, culture of change, and support for staff. Though technology tools facilitated communication and accountability, not all participants embraced technology and some reported challenges with use.

\section{Conclusions:}

Individually tailored HBCR or hybrid CR can facilitate access for patients to participate outside of a CR center. Ongoing research is needed to understand the long-term outcomes of flexible delivery models that may include both in-person and remote visits, and the role of technology in these models.

\section{Introduction}

Cardiac rehabilitation (CR) reduces mortality, morbidity, and hospitalizations. ${ }^{1}$ CR has traditionally been offered in-person at rehab centers where patients receive exercise training, health education, psychosocial management, and tobacco cessation counseling from a multidisciplinary team. A recent scientific statement endorsed home-based CR (HBCR) as an acceptable alternative for some patients. ${ }^{2}$ Increasingly, digital tools (e.g., videoconferencing, mobile applications, and wearables) have augmented the ease of delivering programs outside of the traditional rehabilitation center setting. ${ }^{3,4}$ 
In response to distancing policies during the COVID-19 pandemic, many CR centers suspended in-person services. ${ }^{5}$ Some centers, including the University of California San Francisco (UCSF), pivoted to HBCR as an alternate mechanism of care delivery. Prior studies of HBCR have demonstrated feasibility and efficacy despite few of these including programs with modern videoconferencing. ${ }^{6-8}$ Though many centers anecdotally transitioned to remote care during COVID-19, few have published their experiences, and even fewer have studied their efforts qualitatively. $5,9-12$

In March of 2020, the University of California San Francisco (UCSF) began delivery of HBCR through telephone and video visits with optional use of mobile applications. We sought to qualitatively analyze the experience of patients and staff during the implementation of this program to understand the key barriers and facilitators to implementation.

\section{Methods}

\section{DESIGN \& POPULATION}

We conducted a qualitative evaluation of the implementation of HBCR at UCSF. Patient participants included individuals enrolled in UCSF CR who had at least one telephone or in-person encounter with UCSF CR staff between March 16, 2020 and May 27, 2020. All participants were of adult age and English speaking.

Staff participants included UCSF staff members (e.g., physician, nurses, exercise physiologists, dietician, and administrative staff) providing patient care and operational support to the UCSF Health Cardiac Rehabilitation and Wellness Center as of March $16^{\text {th }}, 2020$.

All participants provided verbal informed consent for this minimal risk study. The study was reviewed and approved by the UCSF Institutional Review and Ethics Board. The methods were performed in accordance with all relevant guidelines and regulations and approved by the ethics committee at UCSF.

\section{INTERVENTION}

To meet the need for CR during COVID-19, UCSF implemented HBCR, which included the core components of $\mathrm{CR}$ and was individually tailored to patients' needs and limitations at home (Figure )..$^{13}$ Patients interacted with CR staff through weekly telephone or video visits for individual exercise plans, health education, and motivational counseling. Patients engaged in 1-hour group wellness sessions focused on emotional wellbeing and health education over telephone or video with other patients and multidisciplinary staff. Patients were referred for telephone or video consultations with nutritionists, pharmacists, mental health professionals, and tobacco cessation counseling as indicated.

Patients were invited to use a free mobile phone application called Better Hearts (Chanl Health), which allowed for logging exercises and vitals, receiving medication reminders, viewing educational materials, and messaging with providers. Patient data was uploaded to a cloud-based server where providers could 
view the data through a patient management dashboard. Additionally, providers could create exercise prescriptions and exchange messages with patients. Patients were also invited to use a separate online platform called Tiatros designed to provide programming for psychosocial resilience and behavioral health support. Patients participated in an 8-week program with a peer group and trained mental health facilitators that included educational videos, learning materials, and psychosocial exercises.

\section{INTERVIEWS}

Patients and staff participated in semi-structured interviews about their experiences with health-related behaviors during COVID-19, HBCR, and technology tools used to facilitate HBCR. A single staff member interviewed participants following an interview guide [Supplement]. Interviews were audio-recorded and transcribed verbatim through an online transcription service (i.e., Rev.com).

\section{QUESTIONNAIRES}

All participants received a unique link to a Qualtrics questionnaire to evaluate Tiatros and Better Heart applications [Supplement]. Participants who were unable to complete the web-based questionnaire completed the questionnaire over the phone, administered by study staff. Responses were summarized using descriptive statistics.

\section{ANALYSIS}

Patient demographics were summarized using descriptive statistics. Interview transcripts were analyzed using a rapid qualitative template analysis which included themes from the Theory of Planned Behavior (e.g., attitudes, beliefs, subjective norms, perceived behavioral control, behavioral intention), Unified Theory of Acceptance and Use of Technology (e.g., performance expectancy, effort expectancy, facilitating conditions, social influence, habit, price value, hedonic motivation, and technology use intention), and Consolidated Framework for Implementation Research [Supplement]. ${ }^{14}$ Templates were iteratively revised to incorporate emergent themes. Two reviewers independently coded each interview and discussed discrepancies to achieve greater than $95 \%$ concordance. Key themes and quotes were extracted and collated for analysis.

\section{Results}

\section{SCREENING AND ENROLLMENT}

From March 16, 2020 to May 27, 2020, 38 potential participants were identified from electronic health records. Of the 38 potential participants, 15 did not respond and 1 was not contacted due to privacy restrictions. Twenty-two participants were contacted, but 5 were ineligible due to a language barrier. Of the 17 eligible participants, 13 consented but 1 withdrew before the interview; ultimately, 12 participated. Participants ranged in age from 48 to 83 (mean 64.8 years). There were 8 females $(66.7 \%)$ and 4 males. There were 8 self-identified white individuals (66.7\%), 2 were Asian (16.7\%), 1 was Black (8.3\%), and 1 
was multi-racial (8.3\%). Staff were all 18-64-years of age and include both men and women of different races and ethnicities (more specific descriptions not reported due to small numbers and privacy protections).

\section{QUESTIONNAIRES}

Of the patient participants, eight (75\%) reported using mobile apps or websites to help them participate in CR. Three participants used the Better Hearts app and responded to questions. Four participants used the Tiatros app and three responded to questions. Five staff members responded to questions about the Better Hearts dashboard [Supplement].

\section{NARRATIVE DESCRIPTION OF CR EXPERIENCE}

Patients highlighted the fear, isolation, and disruption of life activities that resulted from the COVID-19 pandemic.

My big change was to avoid the Coronavirus at all costs and I have been holed up in this apartment since the middle of March. [Patient-9018]

\section{I have been completely isolated and I'm terrified of this pandemic. [Patient-9001]}

I don't want to go back because I'm still afraid to be in the room with other people. [Patient-9019]

I'm afraid to go outside, and the couple times that I have gone outside, there's so many people out there ... a lot aren't wearing any masks and it freaks me out. [Patient-9038]

The effects of the pandemic on health-related behaviors were variable. Some people found behaviors like physical activity easier to maintain, while others found them more difficult.

And ironically, I found more time and more ways to exercise, and I've been eating healthier and less. [Patient-9034]

Previously I got a great deal of exercise, making more frequent trips to the grocery stores and doing more shopping and doing more walking and riding the bus more. So, now that I'm holed up in the place, my exercise has been spotty. [Patient-9018]

Yeah, because I'm home, bored, so I cook. I cook a lot of different meals, different recipes. [Patient-9019]

Participants and staff highlighted the importance of CR to be individually tailored. Many patients praised the adaptability of HBCR.

\section{[About center CR] They would change the music based on the age group. [Patient-9038]}




\section{The staff there really adapted and improvised. [Patient- 9034]}

Home-based cardiac rehab helps individuals focus on how to create that time in their life outside of the center. [Staff-006]

I got a better grasp of how to create a personalized exercise program. I would learn that someone likes dancing, and start looking into Zumba videos, and then it was a success. [Staff-002]

I find out what kinda equipment they have at home. Do they have a blood pressure cuff at home? Do they have a scale at home? Do they have a wearable activity tracker? And build a program based on what they have. [Staff-003]

Some patients preferred in-person sessions, while others preferred the convenience of HBCR, and still others preferred a hybrid mix of in-person and home-based sessions.

The main thing that's wonderful is that I don't have to come up to the hospital. [Patient-9005]

\section{Everything that I get in the Rehab Center I'm getting at, at home. [Patient-9025]}

\section{I much prefer in-person, of course. [Patient-9001] \\ Now, I'm kinda doing both... and I kinda like it that way. [Patient-9029]}

An important facilitator for in-person and HBCR was a strong relationship between participants and staff, who serve as source of accountability. They helped patients "feel safe" and troubleshoot technology.

[About center CR] I was going three times a week and loved it. Everyone there I absolutely adore, and I love the idea of having someone making me feel safe when I tried to do the treadmill. [Patient-9001]

I was very worried I was gonna drop dead and have a heart attack. But they assured me, and with Zoom, even when I do my physical exercise, [CR Staff] are right there with me should something, you know, happen. [Patient-9001]

\section{[About Better Hearts] Okay, I'll do it because you asked me to. [Patient-9029]}

I always feel like he's my personal cheerleader and it's kind of endearing and fun. [Patient-9029]

[About Better Hearts] It helps me to stay accountable, and l'd like to think that it also has helped the cardiac rehab folks, so they have an idea of what my stats are. [9034] 
It has allowed them to certainly minimize their risk and their exposure, gives them peace of mind, allows them to have their family members participate, which has been really interesting and helpful. [Staff-007]

Group telephone and video wellness sessions were praised by patients.

It was very helpful. I would say that it was, at the beginning of shelter in place, this regular routine. The check in was very helpful from not just the physical perspective, but the mental perspective. [9024] We would share each other's experiences and have great conversations. [9003]

Lots of different people, different ages, different walks of life all come together on these- these Tuesday and Friday Zoom chats and compare and contrast. We're all going through very stressful times above and beyond our heart ailments. It's interesting to hear other people's perspective. [9034]

Patients and staff had a range of attitudes toward technology tools.

\section{Another app. [Patient-9038]}

\section{I wanna do everything with a pen and paper. [Patient-9038] \\ Absolutely, not a problem[about using an ap.] [Patient-9034]}

I would like to always do it over Zoom, so if we exercise, I can see what they're doing and give them form correction. [Staff - 003]

\section{I'm not very tech savvy myself. [Staff - 001]}

Digital tools helped patients remain connected with providers and allowed providers to obtain information and monitor progress. However, patients and staff discussed technology adoption challenges including the mismatch between expected and observed performance, ease of use, compatibility with other systems, and the need for training.

[About Better Hearts] I think it's more handy for [CR staff] to see what we were doing. [Patient-9038]

\section{[About Better Hearts] Apps like these and Fitbit keep me more accountable. [Patient-9034]}

[About Tiatros] The whole thing was kinda rolled out, and not a lot of instructions were passed on. And then you're suddenly getting these emails and prompts that said, "Week one has started and you're paired with so-and-so." Well, paired to do what exactly? [Patient-9034]

\section{If Fitbit and the Better Hearts could combine together it would be perfect. [Patient-9024]}


[About Map My Walk] He gave me also an app that I use on my phone that now whenever I go out for a walk I hit the app and just measure the distance. This app is good 'cause it records everything. [9003]

\section{NARRATIVE DESCRIPTION OF HBCR IMPLEMENTATION}

Staff interviews revealed that facilitators to implementing HBCR included a team culture that was conducive to change and supported by leadership.

Because we're in a new cardiac rehab program our directors and our staff were really open to new ideas. [Staff-002]

One of the things that I noticed is that the Cardiac Rehab Team works really well together. Every single person on the team contributes. [Staff-005]

Due to COVID-19, when we shut down, we had to figure out a way to see our patients, and we implemented a home-based cardiac rehab program. [Staff-004]

Really critical was support from administration. [Department leadership] was extremely supportive of the whole project, and also very supportive of the move to home-based cardiac rehab. That was despite the fact that at the beginning, we were not getting reimbursed for home-based cardiac rehab. Having that strong level of administrative support was really important. [Staff-005]

The transition to HBCR, incorporating new delivery models and technology, also required supporting staff with new workflows.

I'm an old nurse and I have just recently had to learn how to document in the electronic health record. For me, the software that the hospital uses is challenging. [Staff-001]

I think it's been a big adjustment for people like me who are not used to working at home. The obstacles that I find are that I sit way too much at the computer. [Staff-001]

\section{My home life has always been completely separate. [Staff- 001]}

Supplying all of us with laptops and IT supports that took some time since none of us had really worked from home before. [Staff-002]

\section{I kind of feel like I'm learning with the patients. [Staff-002]}

In HBCR models, providers helped patients use technology tools and form new habits.

Usually I would have to get on the phone with them and walk them through how to troubleshoot. [Staff007] 
[About Zoom] Make sure that they knew how to click on the access code and what the password was. Most people have been able to pick it up but I know that there's some people who need repetitive information. [Staff-006]

I think having us introduce the app to them here in the center has improved the enrollment. [Staff-002]

[About Better Hearts] What I've had to do is remind people to use the app. [Staff-001]

Patients maybe forget because it's not part of their regular schedule. They're not used to having these type of video visits. [Staff-007]

HBCR may be similar to CBCR, but can also pose new challenges (e.g., privacy, patient access to technology).

Some people just aren't comfortable with video... you have your whole house behind you. You may not want to, it's a personal space. [Staff-004]

It's not as good as when you're able to be hands on and you could take an exercising blood pressure while they're in the middle, and you get to see their EKG the whole time. But other than really getting that diagnostic material, it's pretty close to being the same. [Staff-003]

HBCR may be associated with different costs and reimbursement for centers but may reduce some patient out-of-pocket costs.

\section{It was very cost effective for him because he was catching cabs. [Staff-004]}

\section{We got a lot of support with getting laptops. [Staff- 002]}

Even though we weren't going to be reimbursed we realized the importance of patient care. [Staff - 002] We've created a billing process, so that's been a big hurdle and we'll see if that's successful. [Staff-006]

\section{Discussion}

UCSF rapidly implemented HBCR in response to COVID-19. Our qualitative interviews demonstrate key strengths of HBCR including the ability to deliver an individually tailored program (Table I). Strong relationships with staff who served as a source of accountability and technology support were a key facilitator of patient experience. Similarly, a strong team culture, institutional buy-in, and support for staff were important factors for implementation. Finally, heterogeneous attitudes toward remote care and technology highlight that flexible hybrid delivery models may be needed to meet the varying preferences and needs of patients. The UCSF experience during the COVID-19 pandemic led to a flexible hybrid delivery model in which patients attend an individualized program of in-person and/or remote telephone or video visit sessions (Figure I). 
As CR programs consider how to expand access, HBCR and hybrid programs, enhanced by new digital technology tools, may be an effective strategy. ${ }^{3,15,16}$ Prior studies have shown that HBCR and hybrid CR are not significantly different from traditional CR in terms of outcomes and completion rates. ${ }^{7,17-19}$ Qualitative studies of telerehabilitation outside of the US have reported the importance of individual tailoring and connections between staff and patients for promoting accountability. ${ }^{20-22}$ Studies have also identified the potential barrier of limited peer engagement in remotely delivered CR. ${ }^{20}$ Our study demonstrates that providing telephone or video group wellness sessions may address this barrier. The incorporation of technology tools into CR programs can pose challenges, but emerging evidence and our results suggest that it may be possible to include technology tools that meet expected performance, are easy to use, have appropriate training, and have their use supported by staff. ${ }^{23}$

Organizational factors also contribute to the implementation of new delivery models for CR. Previous work has reported that factors such as leadership support, funding, and institutional contribute to CR delivery. ${ }^{24,25}$ This study adds further qualitative data to support these facilitators, and adds information on organizational factors that contribute to technology adoption for CR programs, including equipment and workflow modifications for CR staff.

Our study has limitations including the small number of participants. However, two reviewers found saturation of major themes on independent review. Further, some sampling bias may exist because patients who participated in interviews may have been more engaged. However, our sample did include one patient who chose not to continue participating in the program. Additionally, our study sample was limited to English-speaking participants. The CR program has delivered HBCR to patients with limited English proficiency with the use of interpreter services, but this was not studied.

Despite the data supporting the benefits of $\mathrm{CR}$, the proportion of eligible patients participating in $\mathrm{CR}$ remains low. ${ }^{26,27}$ Ongoing innovations in delivery of CR may increase participation. This study provides information that may be helpful for programs seeking to implement new models of CR delivery. Further research is needed to determine whether patient-centered, flexible hybrid delivery models can improve access, patient experience, and clinical outcomes. 
Table 1

Recommendations for Implementing HBCR

\section{HBCR Goals Recommendations}

Optimize

patient

experience
- Assess patient preferences and needs to individually tailor the use of in-person and/or remote sessions

- Facilitate strong relationships with staff early and often

- Hold group wellness sessions that allow patients to build community with staff and one another

- Use patient's home environment to create an individualized experience

Optimize implementation
- Gain institutional and leadership buy-in to develop HBCR including identifying key motivations for change (e.g., COVID-19 pandemic, low enrollment rates)

- Support staff in making the transition to new delivery model including providing equipment (e.g., laptops) and adapting workflow

- Develop mechanisms to incorporate regular feedback (e.g., regular staff meetings and town halls)
Incorporate optional digital tools
- Assess patient attitudes and preferences for technology

- Select tools that allow for tracking exercise, tracking vital signs, and a platform for data sharing and messaging with provider care team

- Support patients in troubleshooting and climbing learning curve with new tools

\section{HBCR Goals Recommendations}

Optimize

patient

experience
- Assess patient preferences and needs to individually tailor the use of in-person and/or remote sessions

- Facilitate strong relationships with staff early and often

- Hold group wellness sessions that allow patients to build community with staff and one another

- Use patient's home environment to create an individualized experience

Optimize implementation
- Gain institutional and leadership buy-in to develop HBCR including identifying key motivations for change (e.g., COVID-19 pandemic, low enrollment rates)

- Support staff in making the transition to new delivery model including providing equipment (e.g., laptops) and adapting workflow

- Develop mechanisms to incorporate regular feedback (e.g., regular staff meetings and town halls)
Incorporate optional digital tools
- Assess patient attitudes and preferences for technology

- Select tools that allow for tracking exercise, tracking vital signs, and a platform for data sharing and messaging with provider care team

- Support patients in troubleshooting and climbing learning curve with new tools 


\section{Declarations}

Ethics approval and consent: This study was approved by the ethics and institutional review board at the University of California San Francisco. The review deemed the methods in accordance with all guidelines and regulations and deemed the study to be minimal risk to all participants. Participants all provided verbal consent to informed consent. All authors reviewed and consent to publish this version of the manuscript.

Consent for Publication: No identifiable information of participants are included in this submission. Not Applicable.

Availability of data and materials: All materials described in the manuscript including all relevant data will be available to any scientist wishing to use them for non-commercial purposes upon request from the corresponding author. All data generated or analyzed during this study are included in this published article.

Competing interests: Dr. Beatty was previously employed by Apple Inc. and holds stock in Apple Inc.

\section{Funding:}

UCSF Department of Epidemiology and Biostatistics

UCSF Department of Medicine, Division of Cardiology

\section{Author contributions:}

CT conducted participant interviews. SG and $A B$ conducted qualitative data analysis. SG wrote the draft of the paper. All authors reviewed the manuscript. AB guided and supported the design of the entire project and paper as the senior author.

\section{Acknowledgements:}

Chanl Health provided the Better Hearts program free of charge through June 2020. Tiatros provided the Tiatros program free of charge.

\section{References}

1. Leon AS, Franklin BA, Costa F, et al. Cardiac rehabilitation and secondary prevention of coronary heart disease: An American Heart Assoc. scientific statement from the Council on Clin. Cardiol. (Subcommittee on Exercise, Cardiac Rehabil., and Prevention) and the Council on Nutr., Phys. Activity, and Metab. (Subcommittee on Phys. Activity), in collaboration with the Am. Assoc.100183597100183597 of Cardiovasc. and Pulmonary Rehabil. Circulation. 2005;111(3):369376. doi:10.1161/01.CIR.0000151788.08740.5C 
2. Thomas RJ, Beatty AL, Beckie TM, et al. Home-Based Cardiac Rehabilitation: A Scientific Statement From the American Association of Cardiovascular and Pulmonary Rehabilitation, the American Heart Association, and the American College of Cardiology. Circulation. 2019;140(1):e69-e89. doi:10.1161/CIR.0000000000000663

3. Sandesara PB, Lambert CT, Gordon NF, et al. Cardiac Rehabilitation and Risk Reduction: Time to "rebrand and Reinvigorate." J Am Coll Cardiol. 2015;65(4):389-395. doi:10.1016/j.jacc.2014.10.059

4. Beatty AL, Fukuoka Y, Whooley MA. Using mobile technology for cardiac rehabilitation: a review and framework for development and evaluation. J Am Heart Assoc. 2013;2(6):e000568. doi:10.1161/JAHA.113.000568

5. Besnier F, Gayda M, Nigam A, Juneau M, Bherer L. Cardiac Rehabilitation During Quarantine in COVID-19 Pandemic: Challenges for Center-Based Programs. Arch Phys Med Rehabil. 2020;101(10):1835-1838. doi:10.1016/j.apmr.2020.06.004

6. Richardson CR, Franklin B, Moy ML, Jackson EA. Advances in rehabilitation for chronic diseases: Improving health outcomes and function. BMJ. 2019;365. doi:10.1136/bmj.I2191

7. Anderson L, Sharp GA, Norton RJ, et al. Home-based versus centre-based cardiac rehabilitation. Cochrane Database Syst Rev. 2017;(6). doi:10.1002/14651858.CD007130.pub4

8. Chen YW, Wang CY, Lai YH, et al. Home-based cardiac rehabilitation improves quality of life, aerobic capacity, and readmission rates in patients with chronic heart failure. Med (United States). 2018;97(4). doi:10.1097/MD.0000000000009629

9. Drwal KR, Forman DE, Wakefield BJ, El Accaoui RN. Cardiac Rehabilitation During COVID-19 Pandemic: Highlighting the Value of Home-Based Programs. Telemed e-Health. June 2020:tmj.2020.0213. doi:10.1089/tmj.2020.0213

10. Dalal HM, Taylor RS, Jolly K, et al. The effects and costs of home-based rehabilitation for heart failure with reduced ejection fraction: The REACH-HF multicentre randomized controlled trial. Eur $J$ Prev Cardiol. 2019;26(3):262-272. doi:10.1177/2047487318806358

11. Frederix I, Hansen D, Coninx K, et al. Medium-term effectiveness of a comprehensive internet-based and patient-specific telerehabilitation program with text messaging support for cardiac patients: Randomized controlled trial. J Med Internet Res. 2015;17(7). doi:10.2196/jmir.4799

12. Angell SY, McConnell M V, Anderson CAM, et al. The American Heart Association 2030 Impact Goal: A Presidential Advisory From the American Heart Association. Circulation. 0(0):CIR.0000000000000758. doi:10.1161/CIR.0000000000000758

13. J. BG, A. WM, A. AP, et al. Core Components of Cardiac Rehabilitation/Secondary Prevention Programs: 2007 Update. Circulation. 2007;115(20):2675-2682. doi:10.1161/CIRCULATIONAHA.106.180945

14. Gale RC, Wu J, Erhardt T, et al. Comparison of rapid vs in-depth qualitative analytic methods from a process evaluation of academic detailing in the Veterans Health Administration. Implement Sci. 2019;14(1):11. doi:10.1186/s13012-019-0853-y 
15. Arena R, Williams M, Forman DE, et al. Increasing referral and participation rates to outpatient cardiac rehabilitation: The valuable role of healthcare professionals in the inpatient and home health settings: A science advisory from the american heart association. Circulation. 2012;125(10):13211329. doi:10.1161/CIR.0b013e318246b1e5

16. Gavic AM. Addressing the problem of cardiac rehabilitation program distribution. $J$ Cardiopulm Rehabil. 2005;25(2):85-87. doi:10.1097/00008483-200503000-00007

17. Clark RA, Conway A, Poulsen V, Keech W, Tirimacco R, Tideman P. Alternative models of cardiac rehabilitation: A systematic review. Eur J Prev Cardiol. 2015;22(1):35-74. doi:10.1177/2047487313501093

18. Southard BH, Southard DR, Nuckolls J. Clinical trial of an internet-based case management system for secondary prevention of heart disease. J Cardiopulm Rehabil. 2003;23(5):341-348. doi:10.1097/00008483-200309000-00003

19. Prasada S, Rambarat C, Winchester D, Park K. Implementation and Impact of Home-Based Cardiac Rehabilitation in a Veterans Affair Medical Center. Mil Med. 2020;185(5-6):E859-E863. doi:10.1093/milmed/usz366

20. Dinesen B, Nielsen G, Andreasen JJ, Spindler H. Integration of rehabilitation activities into everyday life through telerehabilitation: Qualitative study of cardiac patients and their partners. $J$ Med Internet Res. 2019;21(4). doi:10.2196/13281

21. Knudsen MV, Laustsen S, Petersen AK, Hjortdal VE, Angel S. Experience of cardiac tele-rehabilitation: analysis of patient narratives. Disabil Rehabil. 2019. doi:10.1080/09638288.2019.1625450

22. Banner D, Lear S, Kandola D, et al. The experiences of patients undertaking a "Virtual" cardiac rehabilitation program. Stud Health Technol Inform. 2015;209(January):9-14. doi:10.3233/978-161499-505-0-9

23. Beatty AL, Magnusson SL, Fortney JC, Sayre GG, Whooley MA. VA FitHeart, a Mobile App for Cardiac Rehabilitation: Usability Study. JMIR Hum factors. 2018;5(1):e3. doi:10.2196/humanfactors.8017

24. Wakefield BJ, Drwal K, Paez M, et al. Creating and disseminating a home-based cardiac rehabilitation program: experience from the Veterans Health Administration. BMC Cardiovasc Disord. 2019;19(1):1-7. doi:10.1186/s12872-019-1224-y

25. Forman DE, Fix GM, McDannold S, et al. Decisive Bearing of Organizational Dynamics on the Application and Success of Hospital-Based Cardiac Rehabilitation. Mayo Clin Proc. 2016;91(7):975977. doi:10.1016/j.mayocp.2016.04.019

26. Ritchey MD, Maresh S, McNeely J, et al. Tracking cardiac rehabilitation participation and completion among Medicare beneficiaries to inform the efforts of a national initiative. Circ Cardiovasc Qual Outcomes. 2020;13(1). doi:10.1161/CIRCOUTCOMES.119.005902

27. Beatty AL, Truong M, Schopfer DW, Shen H, Bachmann JM, Whooley MA. Geographic variation in cardiac rehabilitation participation in medicare and veterans affairs populations: Opportunity for improvement. Circulation. 2018;137(18):1899-1908. doi:10.1161/CIRCULATIONAHA.117.029471 


\section{Figures}

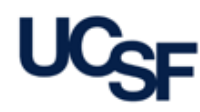

\section{UCSF Cardiac Rehabilitation}

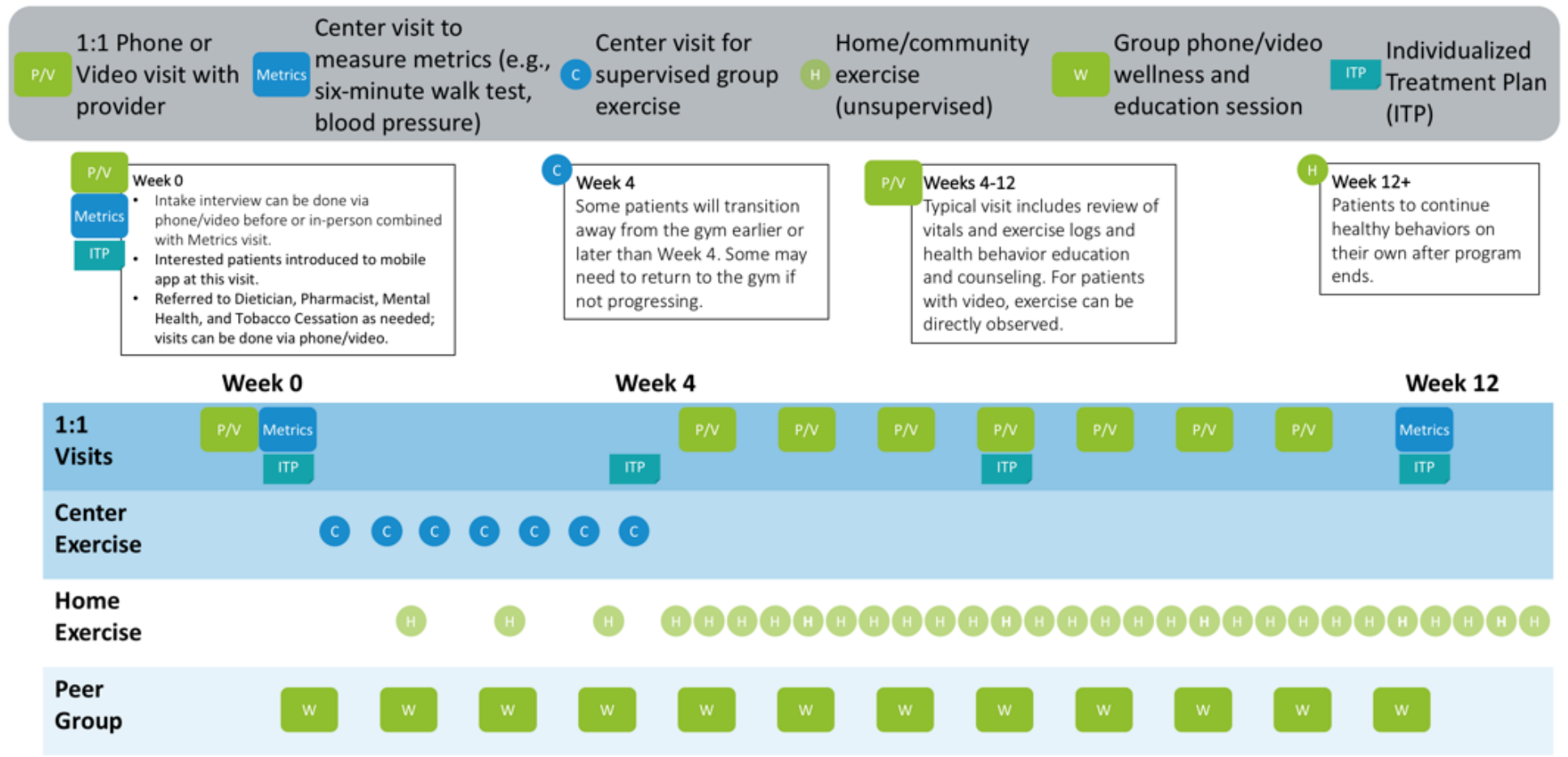

\section{Figure 1}

Diagram of UCSF Cardiac Rehabilitation Program

\section{Supplementary Files}

This is a list of supplementary files associated with this preprint. Click to download.

- SupplementalMaterial.docx 